The High-Resolution Frontier in Infrared Spectroscopy

John H. Black

Department of Earth $\& 5$ Space Sciences

Chalmers University of Technology

Onsala Space Observatory, SE-43992 Onsala, Sweden

This is the preprint version of the introductory chapter of

High Resolution Infrared Spectroscopy in Astronomy

proceedings of an ESO Workshop held in Garching, Germany, 2003

November 18-21

Editors: Hans Ulrich Käufl, Ralf Siebenmorgen, and Alan Moorwood

Published 2005 by Springer (Berlin); ISBN 3-540-25256-8, pages 314

Online version: http://dx.doi.org/10.1007/b96749; see pages 314.

This version: 2004 February 29; updated 2011 October 9

Note: there were two articles directly relevant to suggested observations that appeared too late to be incorporated into the published version.

Ryde, N., Richter, M. J. 2004, ApJ, 611, L41, "Nonthermal MgI emission at 12 micron from Procyon"; and

Banerjee, D. P. K., Ashok, N. M., Launila, O., Davis, C. J., Varricatt, W. P. 2004, ApJ, 610, L29, "A search for radioactive ${ }^{26} \mathrm{Al}$ in the nova-like variable V4332 Sgr", which yielded an upper limit of 0.1 for the 26/27 isotope ratio. 



\title{
The High-Resolution Frontier in Infrared Spectroscopy
}

\author{
John H. Black \\ Chalmers Centre for Astrophysics \& Space Science \\ Onsala Space Observatory, SE-43992 Onsala, Sweden
}

\begin{abstract}
High-resolution astronomical spectroscopy in the infrared from large groundbased telescopes will lead to outstanding developments in many areas of astrophysics. A few examples are discussed, including isotope abundances in rapidly evolving stars, absorption spectroscopy of interstellar clouds, and terrestrial remote sensing with astronomical facilities.

A picture may be worth a thousand words, but a spectrum is worth a thousand pictures - J. S. Miller.

There is no such thing as too much resolution - J. J. Charfman.
\end{abstract}

\section{Defining the Frontiers}

An introduction to a workshop on high-resolution infrared spectroscopy in astronomy should set the stage for both the ideas and the tools to be discussed. To oversimplify the philosophy of science, one school of thought holds that ideas cause major shifts in the ruling paradigms while the other school maintains that scientific progress is mainly driven by tools [1]. It is obvious to the astronomer that both tools and ideas are important. In part, this workshop has been convened to celebrate the tools: the powerful high-resolution spectrographs that have recently gone into operation and the two new instruments, CRIRES and VISIR, that will soon go to the ESO VLT. There is no doubt that the new tools will enlarge our understanding of the universe; however, it is also certain that this will not happen automatically, because the committees that allocate observing time at major observatories must be convinced by ideas.

To define the frontiers for high-resolution spectroscopy in the infrared it is necessary to consider both the advantages of high resolution and the practical trade-offs. High resolution, $\tilde{\nu} / \delta \tilde{\nu} \geq 10^{5}$, directly yields increased sensitivity to weak and narrow spectrum lines compared with spectroscopy at lower resolution for a fixed ratio of signal to noise $(S / N)$ per resolution element. The trade-off is that one must usually sacrifice $S / N$ in going to higher resolution. However there is a further advantage in high resolution for the study of narrow features in an infrared spectrum: the fidelity of corrections for the absorption and emission of Earth's atmosphere will also improve as the resolution approaches the intrinsic widths of the telluric features. Moreover, high resolution can reveal more information from line broadening and line shifts. Line shifts due to the 
Zeeman effect, for example, measure magnetic field strengths in the sources, while Dopper shifts map the kinematics of sources. The infrared region of the spectrum is particularly valuable for investigating cool thermal sources (i.e. temperatures less than a few thousand $\mathrm{K}$ ) as well as any astronomical sources that are highly extinguished by foreground dust. In atmospheres of cool stars and planets, circumstellar envelopes, interstellar matter, and other environments at relatively low temperature, molecules can exist. Molecules are especially attractive spectroscopically for two reasons. First, at high resolution in the infrared, the rotational structure of electronic and/or vibrational bands can usually be resolved, which permits the measurement of rotational population distributions that are sensitive to temperature and density in the source. Second, because rotational and vibrational energies of molecules are sensitive to atomic masses, isotope shifts can be well resolved in molecular spectra while they are often rather smaller than line widths in atomic spectra. Isotope abundances are valuable tracers of nucleosynthetic processes in stars and are important probes of chemical processes in interstellar and circumstellar matter.

The advantages of high-resolution infrared spectroscopy come at some cost in sensitivity, of course. There are additional compromises that must sometimes be considered in the trade-offs among spectroscopic resolution, angular resolution, field of view, and sensitivity. The recent history of infrared spectroscopy of $\mathrm{H}_{2}$ line emission in Orion provides instructive examples of these tradeoffs. Recent observations of the $\mathrm{H}_{2} 1-0 \mathrm{~S}(1)$ line emission at $2.121 \mu \mathrm{m}$ in the Orion Molecular Cloud with a scanning Fabry-Pérot interferometer and adaptive optics [2] reveal exquisite detail in the small-scale structure of the molecular outflow with an angular resolution of $\sim 0.15$ even though the spectroscopic resolution was $\sim 150 \mathrm{~km} \mathrm{~s}^{-1}(R \sim 2000)$. However, the line profiles show considerable kinematical structure when observed at higher velocity resolution $\sim 14 \mathrm{~km} \mathrm{~s}^{-1}$ $(R \sim 21000)$ [3]. In both cases the observations are biased toward the most highly disturbed $\mathrm{H}_{2}$ of highest surface brightness in the 1-0 S(1) line. The assessment of the overall energetics of molecular gas in this part of Orion is considerably different when the $\mathrm{H}_{2}$ line emission is mapped in other transitions over fields of view greater than 1.5 degrees: the powerful shock-excited emission in the starforming core accounts for only $1 \%$ to $2 \%$ of the total power in the 1-0 S(1) when compared with the fluorescent component of $\mathrm{H}_{2}$ on the larger scale [4].

Several examples of research problems are presented in the following sections in order to illustrate the variety of interests that can be served by high-resolution infrared spectroscopy.

\section{Atmospheres of Planets and Planetary Satellites}

Atmospheres of planets have long been investigated by means of high-resolution infrared spectroscopy. Improvements in sensitivity have continued to reveal interesting new aspects of the atmospheres in our solar system. There are also exciting prospects for direct spectroscopic detection of the atmospheres of extrasolar planets with high-resolution infrared techniques. The infrared spectrum of 
Jupiter, for example, shows prominent emission features of $\mathrm{H}_{3}^{+}$arising in its ionosphere and aurorae. This raises the interesting possibility that $\mathrm{H}_{3}^{+}$emission might be detectable in ionospheres of giant exoplanets [5]. Some of the known exoplanets are very close to their parent stars (semi-major axis $a \sim 0.05 \mathrm{AU}$ ). It is very difficult to predict the structures of the neutral atmospheres of such planets; the properties of their ionospheres/magnetospheres are perhaps even less well constrained. Even so, simple models of an ionosphere of the exoplanet HD 209458b at $a=0.045 \mathrm{AU}$ from a sun-like star suggest that the strongest lines of $\mathrm{H}_{3}^{+}$in the $2-4 \mu \mathrm{m}$ wavelength range might have ratios of line intensity to stellar continuum of the order of $10^{-5}$ or higher. It is important to note that at $a=0.045 \mathrm{AU}$, the stellar continuum radiation is so intense that radiative excitation through the $\nu_{2}$ fundamental and overtone bands of $\mathrm{H}_{3}^{+}$will keep the molecule highly excited regardless of the unknown collisional input from hot electrons.

In our own solar system, even the transient atmospheres of planetary satellites can be studied. Recently, de Pater et al. [6] reported the identification of a forbidden band system of SO a ${ }^{1} \Delta \rightarrow \mathrm{X}^{3} \Sigma^{-}$in the infrared spectrum of Io near $1.7 \mu \mathrm{m}$ wavelength. The transient atmosphere of Io owes its existence to outgassing from active volcanoes, although the volcanic products are further modified by interactions with the surface of the satellite and with the magnetosphere of Jupiter. Although SO had previously been observed in this atmosphere at radio frequencies, the new infrared observations reveal a much hotter component of SO molecules, $T_{\text {rot }} \sim 1000 \mathrm{~K}$, which probably trace more directly the volcanic ejecta. Spectra of higher resolution will, of course, afford better diagnostics of the conditions in the volcanic ejecta as well as measurements of the kinematics of the gas. There may also be a valuable synergy with future submillimeter observations with ALMA: the rotational spectrum of $\mathrm{SO} \mathrm{a}^{1} \Delta$ is known and some of the submillimeter lines of SO should be readily detectable [7]. Moreover, ALMA will be able to resolve the $00^{\prime \prime} 1$ disk of Io.

\section{Stellar Atmospheres}

High-resolution infrared spectroscopy of bright stars has provided valuable information on the properties of their atmospheres and on abundances of elements. With the new generation of spectrographs on large telescopes, such investigations can be extended to a greater variety of fainter stars.

\section{1 "Normal" Atmospheres}

The recent observation of $\mathrm{H}_{2} \mathrm{O}$ and $\mathrm{OH}$ in the photosphere of Arcturus shows that even the atmosphere of this well studied star is not fully understood [8]. The unexpectedly strong $\mathrm{H}_{2} \mathrm{O}$ rotational lines appeared in high-resolution spectra at 11 and $12 \mu \mathrm{m}$ wavelength. High resolution $(R=80000)$ was crucial for accurate measurements of the intensities and widths of these lines, which showed that the water must be photospheric rather than circumstellar. The lines of both $\mathrm{H}_{2} \mathrm{O}$ and 
$\mathrm{OH}$ in Arcturus can be explained if the temperature of the outer photosphere is lowered compared with the temperature profiles of standard atmospheric models. However, the ultimate explanation of the unexpected water may have a more fundamental connection with the structures of inhomogeneous atmospheres.

\subsection{Rapidly Evolving Stars}

Although the time-scales of stellar evolution are typically orders of magnitude longer than the lifetimes of human astronomers, there are some stars that change rapidly. These include AGB stars, novae, and supernovae. Isotope abundance ratios can provide exquisite probes of nucleosynthesis and stellar evolution. Because the vibrations and rotations of molecules are sensitive to mass, masssensitive isotope shifts in infrared spectra are readily observable at high resolution. This has been used to advantage in the past to measure relative abundances of isotopes of common elements like carbon and oxygen in normal, bright stars. With more sensitive spectrographs on large telescopes, it should be possible to measure isotope ratios in a broader range of stars and even to observe unstable, radioactive isotopes. Recent observations of nova-like variables suggest an especially valuable experiment in spectroscopic study of rapid stellar evolution. Banerjee et al. [9] have reported the identification of strong emission bands of aluminum monoxide $\mathrm{AlO} \mathrm{A}^{2} \Pi_{i}-\mathrm{X}^{2} \Sigma^{+}$in the infrared spectrum of V4332 Sgr. They suggest that this nova-like, eruptive variable star and a similar object, V838 Mon, may define a new class of "quasi-novae", which eject dust shells, brighten considerably, and then rapidly evolve into much cooler giants or supergiants. In the case of V838 Mon, the great strength of IR bands of $\mathrm{H}_{2} \mathrm{O}$ and $\mathrm{CO}$ led [10] to propose that it is the first identified L-type supergiant. Regardless whether these stars are novae or extremely cool supergiants, it is clear that they exhibit rapid evolution, which includes the formation and ejection of dust. The presence of AlO in the post-eruption spectra of both V4332 Sgr and V838 Mon is particularly intriguing because of the possibility of measuring the abundance of a radioactive isotope, ${ }^{26} \mathrm{Al}$, which has a half-life of $717000 \pm 24000$ years. If the time-scale for mixing between the interior and envelope is smaller than this half-life, then a measurable abundance of ${ }^{26} \mathrm{AlO}$ may be present, and the abundance ratio ${ }^{26} \mathrm{AlO} /{ }^{27} \mathrm{AlO}$ will reflect some interesting details of the nucleosynthetic history of the star. The decay product of ${ }^{26} \mathrm{Al}$ is ${ }^{26} \mathrm{Mg}$, one of the less abundant of the three stable isotopes of magnesium. In a recent study of isotopic ratios of $\mathrm{Mg}$ in giant stars of the globular cluster NGC 6752, [11] found that the abundances of $\mathrm{Al}$ (presumably the stable isotope ${ }^{27} \mathrm{Al}$ ) and of ${ }^{26} \mathrm{Mg}$ are positively correlated with each other even though the relative abundance of ${ }^{26} \mathrm{Mg}$ varies over a wide range. The role of ${ }^{26} \mathrm{Al}$ in an observed anti-correlation of $\mathrm{O}$ and $\mathrm{Al}$ abundances has also been discussed [12].

In the IR spectrum of V4332 Sgr, the A-X $(2,0)$ and $(1,0)$ bands of AlO show the greatest contrast with respect to the continuum, near $1.49 \mu \mathrm{m}$ and $1.67 \mu \mathrm{m}$, respectively. Near the $(2,0)$ band origin, the separations of rotational lines are approximately $\Delta \tilde{\nu} \sim 0.3 \mathrm{~cm}^{-1}$, which means that a resolving power

$$
R \geq \tilde{\nu}_{20} / \Delta \tilde{\nu} \sim 6700 / 0.3 \sim 20000
$$


will be needed to resolve the rotational structure. Because this band has a nonzero vibrational quantum number in the upper state, the isotope splitting is rather larger: approximately $20 \mathrm{~cm}^{-1}$. The $(1,0)$ band is well placed in the 1.65 $\mu \mathrm{m} \mathrm{H}$ photometric band. In 2003 July, V4332 Sgr was fairly bright at $\mathrm{H}=12$ mag. The expected performance of CRIRES would provide a high-resolution spectrum with $S / N \geq 20$ in 1 hour. Note that V4332 Sgr might be confused with V4334 Sgr (widely known as Sakurai's star), which also has an infrared spectrum worth following at high resolution as it evolves rapidly $[13,14]$.

The recent observations of molecular bands in the infrared spectra of novalike variable stars remind us that other bands have been reported in the IR spectra of dust-forming novae, going back to the early work of Ferland et al. [15] on Nova NQ Vul. More recently, Evans et al. [16] observed CO bands in the IR spectrum of Nova Cas 1993 (V705 Cas) and suggested that the CO must play an important role in the cooling of the envelope where dust subsequently forms. Early formation of CO has also been witnessed in Nova Cygni 2001 Number 1 [17]. High-resolution observations would allow the conditions of the moleculecontaining region to be determined more accurately from rotationally resolved spectra of molecules like $\mathrm{CO}$. Higher resolution is also needed in order to retrieve kinematical information from the line profiles. Finally, higher resolution with good $S / N$ would make it possible to search sensitively for other molecules that play a more direct chemical role in the condensation of dust. For example, excess M-band emission in novae prior to the onset of dust formation is usually taken as a signature of the fundamental band of $\mathrm{CO}$; however, there could also be an interesting contribution from $\mathrm{C}_{3}$ in its strong $\nu_{3}$ vibrational band. The ${ }^{13} \mathrm{C} /{ }^{12} \mathrm{C}$ abundance ratio is expected to be a valuable probe of rapid nucleosynthesis in nova explosions; however, it can be measured accurately only in spectra of higher resolution than currently exist. There is also the intriguing possibility of searching for radioactive ${ }^{14} \mathrm{C}$ in $\mathrm{CO}$ in novae, where nucleosynthesis and envelope formation occur on timescales shorter than the half-life, $5700 \pm 30$ years. The ${ }^{14} \mathrm{C}^{16} \mathrm{O}$ molecule has been sought in the circumstellar envelope of the AGB star CW Leo at radio frequencies: a tentative detection [18] was later contradicted by more sensitive observations [19].

Because radioactive decay of ${ }^{26} \mathrm{Al}$ produces both gamma rays and positrons, it is a potential source of ionization of neutral circumstellar envelopes [20]. A possible detection of ${ }^{26} \mathrm{Al}$ in $\mathrm{AlF}$ in $\mathrm{CW}$ Leo has been reported[21]. The abundance of ${ }^{26} \mathrm{Al}$ in mass-loss envelopes of AGB stars is expected to be very sensitive to details of their evolution [22]. Infrared observations of radioactive ${ }^{26} \mathrm{Al}$ in individual sources will be a valuable complement to the gamma-ray line observations of the decay photons at $1.8087 \mathrm{MeV}$, which can now provide maps of the Galactic distribution of the isotope as well as kinematical information from line shapes[23,24].

\subsection{Magnetic Fields in Stars}

The discovery of Rydberg transitions of atomic species in emission in the midinfrared solar spectrum $[25,26]$ offered the possibility of using such Zeeman- 
sensitive lines to investigate magnetic fields in active, late-type stars. The Zeeman sensitivity (ratio of Zeeman shift to thermal Doppler width) of the $12 \mu \mathrm{m}$ $\mathrm{Mg}$ I lines is 5 to 10 times larger than for lines at visible wavelengths, making it possible to measure field strengths as low as $250 \mathrm{G}$ in solar sunspot penumbrae. Wavelength shifts due to the quasi-static Stark effect can also be used to infer electron densities in the line-forming regions [27]. The formation of the $\mathrm{Mg}$ I lines in the solar photosphere has been analyzed in detail [28]. The Mg I $12 \mu \mathrm{m}$ lines were reported in absorption in the spectra of $\alpha$ Ori and $\alpha$ Tau [29]. Although those absorption features in supergiants are more likely due to $\mathrm{H}_{2} \mathrm{O}$, the $\mathrm{Mg} \mathrm{I}$ emission appears clearly in a high-resolution spectrum of $\alpha$ Boo (Arcturus) that was obtained with the TEXES spectrograph [30].

There is also an untested prediction that Mg II Rydberg lines will appear in emission in the IR spectra of B-type stars, notably at 3.09 and $4.76 \mu \mathrm{m}$ [31]. The strong expected limb-brightening may make such lines useful diagnostics of horizontal velocity fields associated with non-radial pulsations.

\section{Pre-Main-Sequence Stars and Their Disks}

The young stellar object HD 141569, a member of the class of Herbig Ae/Be stars, has a very interesting infrared spectrum, with $\mathrm{H}_{3}^{+}$lines in emission [32]. The parallax of HD 141569 is $10.10 \pm 0.83$ mas and its visual magnitude is approximately 7 , hence it is one of the nearer, brighter examples. Li and Lunine have modelled the spectral energy distribution of the double-ring disk in this source with porous dust and PAH molecules [33]. The proposed protoplanetary blob suggested by the $\mathrm{CO}$ and $\mathrm{H}_{3}^{+}$emission has been discussed in the context of the theory of a giant vortex in a disk, which concentrates solid material [34]. Such vortices have been invoked to explain the remarkable eclipse events seen in the pre-main-sequence star V582 Mon (= KH 15D) [35-37]. Recent multicolor coronagraphic imaging of HD 141569 with HST/ACS suggests that the tidal interaction with nearby companions, rather than the formation of a giant planet, is the cause of the unusual structure of the disk [38].

\subsection{Kinematics of Young Stellar Objects}

Jaffe et al. [39] have recently made a dramatic demonstration of the value of high-resolution IR spectroscopy in the study of kinematics of ionized gas in ultra-compact H II regions. Their $\lambda 12.8 \mu \mathrm{m}[\mathrm{Ne}$ II] emission-line maps were made with angular resolution of $1.4 \operatorname{arcsec}$ and velocity resolution $\Delta V=3.4$ $\mathrm{km} \mathrm{s}^{-1}$.

\subsection{Veiled Protostars}

Protostars and many young stellar objects are attended by circumstellar material in the form of extended atmospheres, disks of dust and/or gas, outflows, and jets. Even in the infrared region of the spectrum, it can be difficult to assess the 
nature of the (proto)stellar source because the emission of an associated warm disk can be so intense that it greatly reduces the equivalent widths of absorption features that would otherwise reveal the underlying photosphere. This effect is often called "veiling". In other words, absorption features that would otherwise be rather strong, appear quite weak in relation to the continuous radiation from circumstellar material. Sensitivity to absorption lines of small equivalent width improves directly with resolving power, provided that adequate $S / N$ ratio can be achieved in the continuum. The value of high resolution has been demonstrated in Keck II/NIRSPEC observations of two luminous, Class I protostars in the $\rho$ Ophiuchi star-forming complex [40].

\section{Interstellar Absorption Spectroscopy}

High-resolution infrared absorption spectroscopy has a special role to play in the investigation of interstellar clouds. This technique makes possible the direct measurement of cold molecular hydrogen in regions where this most abundant interstellar molecular would be otherwise invisible. Even though the vibrationrotation lines of $\mathrm{H}_{2}$ occur only as forbidden, electric quadrupole transitions, they are observable weakly in absorption wherever a column density $N\left(\mathrm{H}_{2}\right)>$ $10^{22} \mathrm{~cm}^{-2}$ lies in front of a suitably bright continuum source. Because the larger electric dipole transition strength in the first overtone band of $\mathrm{CO}$ more than compensates for its abundance relative to hydrogen, $\mathrm{CO} / \mathrm{H}_{2} \sim 10^{-4}$, the interstellar absorption lines of $\mathrm{CO}$ in the $\lambda 2 \mu \mathrm{m}$ region are also readily detectable in thick clouds where the cold $\mathrm{H}_{2}$ can be found. Although the lines of the fundamental band of $\mathrm{CO}$ tend to be highly saturated in the spectra of thick clouds, the optical depths of lines of the less abundant isotopomers ${ }^{13} \mathrm{CO}$ and $\mathrm{C}^{18} \mathrm{O}$ are small enough in the fundamental band to be unsaturated but large enough to be detectable. In addition, the vibration-rotation lines of $\mathrm{H}_{3}^{+}$are observable at longer wavelengths in the $\mathrm{L}$ and $\mathrm{M}$ bands. Other non-polar molecules that lack allowed rotational spectra at radio frequencies, like $\mathrm{CH}_{4}, \mathrm{C}_{2} \mathrm{H}_{2}$, and $\mathrm{CO}_{2}$, can be studied in interstellar clouds best (or exclusively) through their infrared-active vibration-rotation bands.

After more than a decade of fruitless searches for the infrared absorption lines of $\mathrm{H}_{2}[41-45]$, the $(1,0) \mathrm{S}(0)$ line at $2.22 \mu \mathrm{m}$ was detected toward NGC 2024 IRS2 by Lacy and collaborators[46]. It has taken another decade to extend these pioneering observations to other sources[47]. The new work is based on spectra at resolving powers $R=45000$ to 75000 obtained with the Phoenix spectrograph on the 2.1 meter and 4 meter telescopes at Kitt Peak. Cold $\mathrm{H}_{2}$ has now been directly observed in 7 thick clouds. The column densities and excitation temperatures derived from the new infrared absorption spectra of NGC 2024 IRS2 are summarized in Table 1.

The directly measured abundance of CO toward NGC 2024 IRS2 is CO/ $\mathrm{H}_{2}=$ $(1.5 \pm 0.2) \times 10^{-4}$. The same abundance is measured toward 6 of the 7 sources with directly observed $\mathrm{H}_{2}$; the abundance toward Elias 29 in the $\rho$ Oph cloud is slightly higher. The interstellar chemistry of $\mathrm{H}_{3}^{+}$is thought to be quite straightforward 
Table 1. Interstellar Absorption Toward NGC 2024 IRS2, Summary of New Results (Kulesa \& Black 2004) [47]

\begin{tabular}{llll}
\hline Molecule & Transitions & $\begin{array}{l}\text { Column Density } \\
\left(\mathrm{cm}^{-2}\right)\end{array}$ & $\begin{array}{l}\text { Excitation Temperature } \\
(\mathrm{K})\end{array}$ \\
\hline${ }^{12} \mathrm{CO}$ & $v=2 \leftarrow 0 \mathrm{R}(0)-\mathrm{R}(8)$ & $(5.9 \pm 0.5) \times 10^{18}$ & $50.0 \pm 0.8$ \\
${ }^{13} \mathrm{CO}$ & $v=1 \leftarrow 0 \mathrm{R}(3)-\mathrm{R}(5)$ & $(1.05 \pm 0.3) \times 10^{17}$ & $47 \pm 3$ \\
$\mathrm{H}_{2}$ & $v=1 \leftarrow 0 \mathrm{~S}(0)[$ para] & $(2.8 \pm 0.2) \times 10^{22}$ & $57 \pm 3$ \\
$\mathrm{H}_{2}$ & $v=1 \leftarrow 0 \mathrm{~S}(1)[$ ortho] & $(1.2 \pm 0.1) \times 10^{22}$ & \\
$\mathrm{H}_{3}^{+}$ & $\nu_{2}=1 \leftarrow 0[$ para] & $(1.5 \pm 0.4) \times 10^{13}$ & $58 \pm 8$ \\
$\mathrm{H}_{3}^{+}$ & $\nu_{2}=1 \leftarrow 0$ [ortho] & $(1.7 \pm 0.5) \times 10^{13}$ & \\
\hline
\end{tabular}

in dense molecular clouds [48-50]. The rate of formation of $\mathrm{H}_{3}^{+}$is limited by the cosmic-ray ionization rate of $\mathrm{H}_{2}$, which can be expressed in terms of an ionizing frequency $\zeta_{0} \mathrm{~s}^{-1}$, which includes secondary processes. The observed $\mathrm{CO}$ abundance can be used to estimate the rate of destruction of $\mathrm{H}_{3}^{+}$in such a molecular cloud. The ionizing frequency can then be determined from the observed abundance ratio $\mathrm{H}_{3}^{+} / \mathrm{H}_{2}$. Toward NGC 2024 IRS2 a value $\zeta_{0}=(3.3 \pm 1.2) \times 10^{-17} \mathrm{~s}^{-1}$ is derived [47]. This ionizing frequency is consistent with expectations. The observed fact that the excitation temperatures of the ortho and para spin species of $\mathrm{H}_{2}$ and $\mathrm{H}_{3}^{+}$are equal to each other and to the $\mathrm{CO}$ rotational temperature supports the idea that the lowest rotational states of $\mathrm{H}_{2}$ and $\mathrm{H}_{3}^{+}$are well thermalized by reactive collisions $\left(\mathrm{H}_{2}\right.$ with $\mathrm{H}^{+}$and $\mathrm{H}_{3}^{+} ; \mathrm{H}_{3}^{+}$with $\left.\mathrm{H}_{2}\right)$.

In diffuse molecular clouds where the electron fraction $e^{-} /\left(\mathrm{H}+\mathrm{H}_{2}\right) \sim 10^{-4}$, $\mathrm{H}_{3}^{+}$is removed by electrons much faster than in the dark clouds described above. As a result, $\mathrm{H}_{3}^{+}$was thought to be undetectable in diffuse clouds. The discovery of $\mathrm{H}_{3}^{+}$in diffuse clouds was a surprise and its unexpectedly high abundance constitutes a crisis for interstellar chemistry [51-55]. The abundances of two other molecular ions in diffuse molecular clouds, $\mathrm{CH}^{+}$and $\mathrm{HCO}^{+}$, continue to lack adequate explanation.

Infrared spectroscopy offers the possibility to observe molecules in both gaseous and solid form in the same absorbing columns. Such studies have already revealed important insights into the chemical evolution of star-forming regions [56]. The solid-phase absorption features arising in ice mantles on interstellar dust particles are intrinsically broad and can be studied to advantage at low resolution [57]. Low-resolution spectra, $R \leq 1500$, obtained with spectrometers on the Infrared Space Observatory (ISO), can even reveal significant components of high-temperature molecular gas; however, such spectra cannot provide accurate determinations of the temperatures and column densities of the predominant colder components. For example, our [47] high-resolution observations of the CO line absorption in the $v=2 \leftarrow 0$ band toward Elias 29 in the $\rho$ Oph cloud are dominated by a cold component of small velocity dispersion (Doppler parameter $b=1.6 \mathrm{~km} \mathrm{~s}^{-1}$ ) where the rotational temperature $T_{\text {rot }}=28 \mathrm{~K}$ and total column density $N(\mathrm{CO})=(6.4 \pm 0.4) \times 10^{18} \mathrm{~cm}^{-2}$. This 
component is not recognized in the ISO observations of CO in the $v=1 \leftarrow 0$ fundamental band [58], which clearly reveal a hot component of $\mathrm{CO}$ but cannot constrain its properties accurately.

The study of gas-phase interstellar chemistry stands to benefit from highresolution spectroscopy, especially in the mid-infrared $(\lambda \geq 3 \mu \mathrm{m})$ where it is possible to observe several crucial, non-polar molecules such as $\mathrm{CH}_{4}, \mathrm{C}_{2} \mathrm{H}_{2}, \mathrm{CH}_{3}$, $\mathrm{NH}_{3}$, and $\mathrm{CO}_{2}$, as well as polar molecules like $\mathrm{HCN}$ [59]. An important chemical issue that deserves further attention concerns the remarkably high abundances of multiply deuterated molecules in dense molecular clouds: $\mathrm{D}_{2} \mathrm{CO}, \mathrm{NHD}_{2}, \mathrm{ND}_{3}$, $\mathrm{CHD}_{2} \mathrm{OH}$, and $\mathrm{D}_{2} \mathrm{~S}[60-65]$. Although it can be argued that $\mathrm{D}_{2} \mathrm{CO}$ and $\mathrm{CHD}_{2} \mathrm{OH}$ are enhanced through reactions in ice mantles on cold dust grains, the abundances of the deuterated forms of ammonia have been explained satisfactorily in terms of gas-phase fractionation processes in regions where there is severe depletion of heavy elements. As stressed by Parise et al. [64], the interpretation of isotope fractionation is sensitive to fundamental questions about the chemical roles of interstellar dust and gas-dust interactions. In gas-phase schemes, some of the deuterium enrichment takes place through temperature-sensitive isotopeexchange reactions involving $\mathrm{H}_{3}^{+}, \mathrm{H}_{2} \mathrm{D}^{+}$, and $\mathrm{HD}_{2}^{+}$. Indeed, chemical models suggest that the abundance of $\mathrm{HD}_{2}^{+}$can be similar to that of $\mathrm{H}_{2} \mathrm{D}^{+}$in dense, depleted regions [66]. This suggests a valuable role for infrared spectroscopy: there will be potential interstellar absorption lines of $\mathrm{HD}_{2}^{+}$arising in its two lowest rotational levels at wavelengths near 3.6, 4.6, and $5.0 \mu \mathrm{m}$. The strongest ground-level transitions of the $\nu_{3}$ fundamental band at $\tilde{\nu}=2118.6$ and 2159.1 $\mathrm{cm}^{-1}$ are flanked by low- $J$ lines of ${ }^{12} \mathrm{CO}$ and ${ }^{13} \mathrm{CO}$; therefore, there may already exist sensitive astronomical spectra that include these lines. The frequency of the $\mathrm{HD}_{2}^{+} 1_{10}-1_{01}$ pure rotational transition at $691.66 \mathrm{GHz}$ has recently been measured in the laboratory [67], so there is the possibility of applying complementary infrared and submillimeter techniques to the search for this ion.

\section{Spectroscopy of Extragalactic Sources}

At low and medium resolution, $\tilde{\nu} / \delta \tilde{\nu} \leq 3000$, infrared spectroscopy of galaxies has been extremely valuable. For example, the K-band spectra of starbursts offer diagnostic probes of ionized gas ( $\mathrm{H} \mathrm{I} \mathrm{Br} \gamma)$, excited molecular gas $\left(\mathrm{H}_{2}\right.$ vibrationrotation lines), and the youngest stellar population (photospheric CO bands in red supergiants). The K-band spectra of active galactic nuclei (AGN) frequently show strong $\mathrm{H}_{2}$ line emission and mid-infrared spectra provide an excellent way to separate the contributions of AGN and starbursts to the central luminosities of many active galaxies. For the most part, such work does not require high resolution (e.g. [68]); however, there are fascinating possibilities for highresolution infrared spectroscopy together with adaptive optics systems on large telescopes. For example, absorption spectroscopy at radio frequencies has revealed the presence of neutral hydrogen and even a tentative detection of $\mathrm{CO}^{+}$ in the central obscuring torus of the compact AGN Cygnus A [69,70]. Because the total column density of hydrogen implied by X-ray absorption measurements 
is $N_{\mathrm{H}}>10^{23} \mathrm{~cm}^{-2}$, there might be strong features in the infrared spectrum of the nucleus, including lines of $\mathrm{H}_{3}^{+}, \mathrm{CO}$ and even $\mathrm{H}_{2}$. On the other hand, such observations would push the limits of the largest telescopes: the $2 \mu \mathrm{m}$ brightness of Cygnus $\mathrm{A}$ is $\mathrm{K}=18.5$ at 0 '! 1 angular resolution.

\section{Back to Earth}

One thing that adds to the difficulty of near-infrared spectroscopy at groundbased telescopes is the terrestrial airglow. The airglow emission lines of $\mathrm{OH}$ are an unwanted source of noise for astronomers. It is worth pointing out that this airglow spectrum provides valuable information about Earth's lower thermosphere. Because the $\mathrm{OH}$ airglow emission arises mainly as a result of a specific chemical process

$$
\mathrm{O}_{3}+\mathrm{H} \rightarrow \mathrm{OH}^{*}+\mathrm{O}_{2}
$$

the airglow photons record the deaths - by natural causes - of ozone molecules at an altitude around $90 \mathrm{~km}$. At intermediate resolution, the relative intensities of these $\mathrm{OH}$ lines yield directly the temperature at this level in the atmosphere and at higher resolution there may be additional information about kinematics of high-altitude ozone and hydroxyl.

Astronomical spectrographs have long been used for coincidental investigations of the the terrestrial atmosphere, most recently at visible and far-red wavelengths by Osterbrock and collaborators [71-76]. In the near future, valuable aeronomical information might be retrieved from archives of high-resolution astronomical spectra in the infrared, too.

Low-resolution infrared spectra have recently revealed the production of $\mathrm{BrO}$ in the gaseous ejecta of terrestrial volcanoes, thus apparently solving the mystery of missing atmospheric sources of $\mathrm{Br}$ [77]. A more sensitive survey of volcanic constituents could surely be done with a high-resolution spectrograph of the kind available to astronomers. It is interesting to speculate on the reaction of the European Southern Observatory to a target-of-opportunity proposal to measure high-resolution spectra of a terrestrial volcano the next time a volcanic plume graces the horizon at Cerro Paranal!

\section{References}

1. F. Dyson, "Clockwork Science": New York Review of Books, vol. L, no. 17, pp. 42-44 (2003)

2. M. Gustafsson, L. E. Kristensen, Y. Clénet, D. Field, J. L. Lemaire, G. Pineau des Forêts, D. Rouan, E. Le Coarer: A\&A 411, 437 (2003)

3. A. Chrysostomou, M. G. Burton, D. J. Axon, P. W. J. L. Brand, J. H. Hough, J. Bland-Hawthorn, T. R. Geballe: MNRAS 289, 605 (1997)

4. M. L. Luhman, D. T. Jaffe, L. D. Keller, S. Pak: ApJ 436, L185 (1994)

5. S. Miller et al.: Phil. Trans. R. Soc. Lond. A 358, 2485 (2000)

6. I. de Pater, H. Roe, J. R. Graham, D. F. Strobel, P. Bernath: Icarus 156, 296 (2002) 
7. K. Torstensson, P. Aho: Master's Thesis, Chalmers University of Technology, Göteborg, Sweden (2004)

8. N. Ryde, D. L. Lambert, M. J. Richter, J. H. Lacy: ApJ 580, 447 (2002)

9. D. P. K. Banerjee, W. P. Varricatt, N. M. Ashok, O. Launila: ApJ 598, L31 (2003)

10. A. Evans, T. R. Geballe, M. T. Rushton, B. Smalley, J. Th. van Loon, S. P. S. Eyres, V. H. Tyne: MNRAS 343, 1054 (2003)

11. D. Yong, D. L. Lambert, I. I. Ivans: ApJ 599, 1357 (2003)

12. P. A. Denissenkov, A. Weiss: ApJ 559, L115 (2001)

13. S. P. S. Eyres, A. Evans, T. R. Geballe, A. Salama, B. Smalley: MNRAS 298, L37 (1998)

14. H. U. Käufl, J. Koller, F. Kerber: A\&A 406, 981 (2003)

15. G. J. Ferland, D. L. Lambert, H. Netzer, D. N. B. Hall, S. T. Ridgway: ApJ 227, 489 (1979)

16. A. Evans, T. R. Geballe, J. M. C. Rawlings, A. D. Scott: MNRAS 282, 1049 (1996)

17. R. J. Rudy, et al.: ApJ 596, 1229 (2003)

18. G. A. Wright: ApJ 436, L157 (1994)

19. M. Forestini, M. Guélin, J. Cernicharo: A\&A 317, 883 (1997)

20. A. E. Glassgold: ApJ 438, L111 (1995)

21. M. Guélin, M. Forestini, P. Valiron, L. .M. Ziurys, M. A. Anderson, J. Cernicharo, C. Kahane: A\&A 297, 183 (1995)

22. N. Mowlavi, G. Meynet: A\&A 361, 959 (2000)

23. R. Diehl, F. X. Timmes: PASP 110, 637 (1998)

24. K. Kretschmer, R. Diehl, D. H. Hartmann: A\&A 412, L47 (2003)

25. J. W. Brault, R. Noyes: ApJ 269, L61 (1983)

26. E. S. Chang, R. W. Noyes: ApJ 275, L11 (1983)

27. D. Hoang-Binh: A\&A 286, 607 (1994)

28. J. H. M. J. Bruls, S. K. Solanki, R. J. Rutten, M. Carlsson: A\&A 293, 225 (1995)

29. D. E. Jennings, D. Deming, G. R. Wiedemann, J. J. Keady: ApJ 310, L39 (1986)

30. J. H. Lacy, M. J. Richter, T. K. Greathouse, D. T. Jaffe, Q. Zhu: PASP 114, 153 (2002)

31. T. A. A. Sigut, J. B. Lester: ApJ 461, 972 (1996)

32. S. D. Brittain, T. W. Rettig: Nature 418, 57 (2002)

33. A. Li, J. I. Lunine: ApJ 594, 987 (2003)

34. C. de La Fuente Marcos, R. de La Fuente Marcos: New Astron. 8, 401 (2003)

35. K. E. Kearns, W. Herbst: AJ 116, 261 (1998)

36. P. Barge, M. Viton: ApJ 593, L117 (2003)

37. C. M. Hamilton, W. Herbst, R. Mundt, C. A. L. Bailer-Jones, C. M. Johns-Krull: ApJ 591, L45 (2003)

38. M. Clampin, et al.: AJ 126, 385 (2003)

39. D. T. Jaffe, Q. Zhu, J. H. Lacy, M. Richter: ApJ 596, 1053 (2003)

40. T. P. Greene, C. J. Lada: AJ 124, 2185 (2002)

41. S. G. Kleinmann, D. N. B. Hall, S. T. Ridgway, E. L. Wright: AJ 83, 373 (1978)

42. D. N. B. Hall, S. G. Kleinmann, S. T. Ridgway, F. C. Gillett: ApJ 223, L47 (1978)

43. N. Scoville, S. G. Kleinmann, D. N. B. Hall, S. T. Ridgway: ApJ 275, 201 (1983)

44. J. H. Black, S. P. Willner: ApJ 279, 673 (1984)

45. J. H. Black, E. F. van Dishoeck, S. P. Willner, R. C. Woods: ApJ 358, 459 (1990)

46. J. H. Lacy, R. Knacke, T. R. Geballe, A. T. Tokunaga: ApJ 428, L69 (1994)

47. C. A. Kulesa, J. H. Black: to be submitted (2004)

48. T. R. Geballe: Phil. Trans. R. Soc. Lond. A 358, 2503 (2000)

49. J. H. Black: Phil. Trans. R. Soc. Lond. A 358, 2515 (2000) 
50. E. Herbst: Phil. Trans. R. Soc. Lond. A 358, 2523 (2000)

51. B. J. McCall, T. R. Geballe, K. H. Hinkle, T. Oka: Science 279, 1910 (1998)

52. T. R. Geballe, B. J. McCall, K. H. Hinkle, T. Oka: ApJ 510, 251 (1999)

53. B. J. McCall et al.: ApJ 567, 391 (2002)

54. B. J. McCall et al.: Nature 422, 500 (2003)

55. M. Goto et al.: Publ. Astron. Soc. Japan 54, 951 (2002)

56. E. F. van Dishoeck, G. A. Blake: Ann. Rev. Astron. Astrophys.36, 317 (1998)

57. E. L. Gibb, D. C. B. Whittet, A. C. A. Boogert, A. G. G. M. Tielens: ApJS 151 $35(2004)$

58. A. C. A. Boogert et al.: A\&A 360, 683 (2000)

59. C. Knez, J. H. Lacy, N. J. Evans II, M. J. Richter, A. M. S. Boonman, E. F. van Dishoeck: Rev. Mex. AA 18, 45 (2003).

60. C. Ceccarelli, A. Castets, L. Loinard, et al.: A\&A 338, L43 (1998)

61. E. Roueff, S. Tiné, L. H. Coudert, et al.: A\&A 354, L63 (2000)

62. F. F. S. van der Tak, P. Schilke, H. S. P. Müller, et al.: A\&A 388, L53 (2002)

63. D. C. Lis, E. Roueff, M. Gerin, et al.: ApJ 571, L55 (2002)

64. B. Parise, C. Ceccarelli, A. G. G. M. Tielens, et al.: A\&A 393, L49 (2002)

65. C. Vastel, T. G. Phillips, C. Ceccarelli, J. Pearson: ApJ 593, L97 (2003)

66. H. Roberts, E. Herbst, T. J. Millar: ApJ 591, L41 (2003

67. T. Hirao, T. Amano: ApJ 597 L85 (2003)

68. R. Genzel, C. J. Cesarsky: Ann. Rev. Astron. Astrophys. 38, 761 (2000)

69. J. E. Conway, P. R. Blanco: ApJ 449, L131 (1995)

70. A. Fuente, J. H. Black, J. Martín-Pintado, et al.: ApJ 545, L113 (2000)

71. T. G. Slanger, D. E. Osterbrock: Eos 79, 149 (1998)

72. D. E. Osterbrock, J. P. Fulbright, A. R. Martel, et al.: PASP 108, 277 (1996)

73. D. E. Osterbrock, J. P. Fulbright, T. A. Bida: PASP 109, 614 (1997)

74. D. E. Osterbrock, J. P. Fulbright, P. C. Cosby, T. A. Barlow: PASP 110, 1499 (1998)

75. T. G. Slanger, D. E. Osterbrock: J. Geophys. Res. 105, D1, 1425 (2000)

76. T. G. Slanger, D. L. Huestis, P. C. Cosby, T. A. Bida: Adv. Space Res. 27, 1135 (2001)

77. N. Bobrowski, G. Hönninger, B. Galle, U. Platt: Nature 423, 273 (2003) 